\title{
Discriminação entre olhos normais e glaucomatosos mediante polarimetria de varredura a laser
}

\author{
Discrimination between normal and glaucomatouseyes by scanninglaser polarimetry
}

\author{
Leopoldo Magacho ${ }^{1}$ \\ Ana Maria Marcondes ${ }^{2}$ \\ Vital Paulino Costa ${ }^{3}$
}

\begin{tabular}{|l|}
\hline RESUMO \\
\hline Objetivos: Avaliar a capacidade da polarimetria de varredura a laser de \\
discriminar entre olhos normais e glaucomatosos. Métodos: Cento e doze \\
pacientes com glaucoma primário deângulo aberto e 88 indivíduos normais \\
foram incluídos no estudo. Todos os indivíduos foram submetidos a exame \\
oftalmológico completo, perimetria computadorizada(Humphrey 24-2, Full \\
threshold), avaliando-se Mean Deviation(MD) e Correct Pattern Standard \\
Deviation(CPSD) e exame com GDx. Curvas ROC foram criadas e novos \\
pontos de corte para cada parâmetro individual do GDx estabelecidos para \\
estabelecer uma melhor relação sensibilidade/especificidade (Se/Es) no \\
diagnóstico do glaucoma. A seguir, uma função discriminante com 2 \\
parâmetros do GDx (ellipse modulation e the number) e idade por meio da \\
análise logística multivariada foi criada com o mesmo propósito. Resulta- \\
dos: Osparâmetros individuais do GDx com melhor relação Se/Es foram: the \\
number(Se: 79,5\%, Es: 81,8\%, área abaixo da curva ROC:0,870), maximum \\
modullation(Se: 83,0\%, Es: 76,1\%, área abaixo da curva ROC:0,842)eellipse \\
modulation (Se: 65,2\%, Es: 88,6\%, área abaixo da curva ROC: 0,831). A \\
função discriminante criada obteve resultados superiores a qualquer \\
parâmetro do GDx isolado (Se: 85,7\%, Es: 90,9\%, a área abaixo da curva \\
ROC: 0,920). Conclusão: A combinação de dois ou mais fatores em uma \\
função logística multivariada aumenta a capacidade da polarimetria de \\
varredura a laser de discriminar entre olhos normais e glaucomatosos.
\end{tabular}

Descritores: Disco óptico; Glaucoma/diagnóstico; Perimetria; Lasers; Técnicas de diagnóstico oftalmológico
${ }^{1}$ Coordenador do Setor de Glaucoma do Centro de Referência em Oftalmologia - CEROF, da Universidade Federal de Goiás. Pós-Graduando em Oftalmologia pela Universidade Estadual de Campinas - UNICAMP. 2 Professora Doutora e Chefe do Setor de Neuroftalmologia da Universidade Estadual de Campinas - UNICAMP. ${ }^{3}$ Chefe do Setor de Glaucoma da Universidade Estadual de Campinas - UNICAMP e Professor Livre-Docente da Universidade de São Paulo - USP.

Endereço para correspondência: Leopoldo Magacho, Rua 131 número 313, Setor Sul - Goiânia (GO)

CEP 74093-200

E-mail: lmagacho@brturbo.com

Recebido para publicação em 25.04.2003

Versão revisada recebida em 08.08.2003

Aprovação em 18.11.2003

Nota Editorial: Pela análise deste trabalho e por sua anuência na divulgação desta nota, agradecemos ao Dr. Francisco Eduardo Lopes de Lima.

\section{INTRODUÇÃO}

O glaucoma é caracterizado por danos irreversíveis ao disco óptico e camada de fibras nervosas da retina (CFNR). O defeito perimétrico brancobranco ainda é considerado como "gold standard" no diagnóstico do glaucoma. Entretanto, o aparecimento da lesão é tardia, e só ocorre após a perda de $30 \%$ ou mais de fibras nervosas ${ }^{(1-2)}$. Como o dano glaucomatoso é irreversível, o diagnóstico e tratamento precoces seriam a melhor forma de combate ao glaucoma.

Algumas estratégias de detecção precoce do glaucoma baseiam-se na análise da CFNR, que é precocemente lesada na patogênese da doença ${ }^{(3-4)}$. O exame de polarimetria de varredura a laser faz diversas medidas da espessura da CFNR baseado nas suas propriedades birrefringentes, e tem apresentado bons resultados na detecção precoce do glaucoma ${ }^{(5-9)}$. Além disso, possui reprodutibilidade satisfatória ${ }^{(10)}$ e correlação com o dano funcional no glaucoma (medido através do campo visual) ${ }^{(11-12)}$.

Entretanto, devido à importante sobreposição dos parâmetros indivi- 
duais entre olhos normais e glaucomatosos, tem-se proposto o uso de duas ou mais variáveis em uma fórmula discriminante para aumentar a relação sensibilidade/especificidade (Se/Es) do exame $^{(13)}$. Estudos anteriores mostraram melhora da eficácia do exame ao se construir funções lineares discriminantes ${ }^{(6-7)}$. O nosso estudo tem o objetivo de criar uma função discriminante para a polarimetria de varredura a laser através da regressão logística multivariada, método ainda não utilizado na literatura mundial.

\section{MÉTODOS}

Esse foi um estudo prospectivo comparativo. Após aprovação do Comitê de Ética da Universidade de Campinas (UNICAMP), todos os indivíduos selecionados foram submetidos a exame oftalmológico completo, que incluiu biomicroscopia detalhada, tonometria de aplanação (Goldmann), biomicroscopia de fundo de olho (retina e disco óptico) após midríase, campimetria computadorizada (Humphrey Visual Field Analyser II, programa 24-2, estratégia full threshold, Humphrey Systems, Dublin, CA, USA) e polarimetria de varredura a laser com o GDx (Laser Diagnostic Technologies, San Diego, CA, USA), software 1.0.12. Apenas um olho por paciente foi aleatoriamente escolhido se ambos satisfizessem os critérios de inclusão. Os indivíduos foram consecutivamente divididos em dois grupos: glaucoma e normais.

Os critérios de inclusão para ambos os grupos foram: acuidade visual $\geq 20 / 30$, equivalente esférico $\leq 5$ dioptrias, diâmetro pupilar acima de $2 \mathrm{~mm}$ e dois exames confiáveis de campo visual (perda de fixação $<20 \%$, e falso positivos e negativos < $33 \%)^{(14)}$. Os critérios de exclusão (ambos os grupos) incluíram: história de qualquer doença sistêmica ou ocular (exceto glaucoma) que pudesse causar alterações no exame de perimetria computadorizada ou GDx, cirurgia refrativa, olhos afácicos ou pseudofácicos e catarata importante ${ }^{(15)}$, que pudesse interferir nos exames de campo visual ou de imagem.

Os indivíduos normais foram recrutados entre os membros da Universidade, voluntários da equipe médica, familiares e amigos dos pacientes. Esses foram excluídos se apresentassem pressão intra-ocular (Pio) acima de $21 \mathrm{mmHg}$, disco óptico suspeito (perda localizada da rima, hemorragia de disco óptico, assimetria de escavação $>0,2$, escavação $\geq 0,6$ ) ou campo visual alterado (definido abaixo).

Pacientes com glaucoma foram recrutados do ambulatório da especialidade da UNICAMP. Os critérios de inclusão para esse grupo foram: diagnóstico clínico de glaucoma primário de ângulo aberto (ou seja, duas ou mais medidas da Pio acima de 21 mmHg, gonioscopia demonstrando ângulo aberto, e lesão característica de disco óptico, definido como a presença de pelo menos dois dos seguintes critérios: escavação $\geq 0,6$, perda localizada da rima, hemorragia de disco óptico ou assimetria de escavação $>0,2$ ). Além disso, a presença de pelo menos dois campos visuais confiáveis com perda glaucomatosa característica, definido pelos critérios propostos por Hodapp-Parrish-Anderson ${ }^{(16)}$, eram necessários. Apenas o último campo visual foi considerado para efeito de análise.
Os pacientes glaucomatosos foram classificados pela severidade da lesão de campo visual de acordo com uma modificação dos critérios propostos por Weinreb et $\mathrm{al}^{(6)}$ : a) dano inicial: Mean Deviation (MD) melhor ou igual que -6dB, Corrected Pattern Standard Deviation (CPSD) melhor que 1\% e pelo menos $50 \%$ dos pontos testados dentro dos limites de normalidade; b) dano moderado: MD entre $-6 \mathrm{~dB}$ e $-15 \mathrm{~dB}$ e CPSD pior que $1 \%$; c) dano avançado: MD pior que $-15 \mathrm{~dB}$ ou CPSD pior que $1 \%$.

Para interpretação do exame do GDx, 3 imagens consecutivas e independentes a $15^{\circ}$ foram obtidas, e uma imagem média criada a partir das imagens individuais. Para ser incluída, cada imagem deveria passar pelos critérios de qualidade da máquina. Não houve dilatação pupilar nos pacientes examinados e as luzes do ambiente foram mantidas acesas. Todas as aquisições foram realizadas por um examinador experiente (LM), que delineou as margens do disco óptico de cada paciente. Os parâmetros do GDx e sua significância encontram-se detalhados em publicações prévias ${ }^{(6,17)}$.

A análise estatística foi realizada através do "software" Statistical Analysis System para Windows, versão 8.1 (SAS Institute Inc., Cary, NC, USA). A verificação da normalidade das variáveis foi realizada através do teste de KomogorovSmirnov. Para a estatística descritiva calculou-se a média e o desvio padrão para cada parâmetro do GDx, e a diferença entre indivíduos normais e pacientes glaucomatosos obtida utilizando-se o Teste de T de Student Independente para variáveis com distribuição normal e o Teste U de Mann-Whitney quando os valores assumiram disposição não Gaussiana. Valores de $\mathrm{p}<0,025$ foram considerados como significativos após correção do erro alfa ${ }^{(18)}$. Para dados categóricos foi utilizado o Teste de Qui-Quadrado ou o Teste Exato de Fisher. Curvas ROC (Receiver operator characteristic) foram criadas para cada parâmetro individual do GDx. A área sobre cada curva foi calculada, e novos pontos de corte criados para as variáveis do GDx para melhorar a relação Se/Es. Finalmente, a análise regressiva logística multivariada foi utilizada para criar uma função discriminante com 2 ou mais variáveis do GDx. Esse método tem a mesma finalidade da função linear, com a vantagem de poder ser utilizado com dados não Gaussianos e categóricos.

\section{RESULTADOS}

Um total de 200 indivíduos foi incluído no estudo, 88 normais e 112 glaucomatosos. Em relação à severidade da lesão glaucomatosa, 36 (32,1\%) pacientes foram classificados como glaucoma inicial, 47 (42,0\%) como moderado e 29 (25,9\%) como glaucoma avançado. Os dados demográficos e de campo visual estão ilustrados na tabela 1 .

Ao se comparar os parâmetros do GDx para indivíduos normais e glaucomatosos, verificamos que apenas o parâmetro simmetry não apresentou diferença estatisticamente significante entre os grupos $(\mathrm{p}=0,4)$ (Tabela 2). Os parâmetros do GDx que apresentaram melhor Se/Es na detecção do glaucoma foram: the number (Se: 79,5\%, Es: 81,8\%, área abaixo da curva 
ROC: 0,870), maximum modulation (Se: 83,0\%, Es: 76,1\%, área abaixo da curva ROC: 0,842) e ellipse modulation (Se: 65,2\%, Es: 88,6\%, área abaixo da curva ROC: 0,831) (Tabela 3). Entretanto, podemos constatar considerável superposição para parâmetros individuais do GDx entre olhos normais e glaucomatosos, mesmo para as variáveis com maior capacidade de discriminação entre os grupos (Figura 1).

Finalmente, através da análise de regressão logística multivariada, e pelo processo de seleção de variáveis "stepwise", obtivemos a seguinte fórmula discriminante com os parâmetros the number, ellipse modulation e idade:

$\pi(x)=\frac{\exp (-5,49+0,10 * \text { idade }-0,69 * \text { ellipse }+0,04 * \text { number })}{1+\exp (-5,49+0,10 * \text { idade }-0,69 * \text { ellipse }+0,04 * \text { number })}$

Com o ponto de corte $\geq 0,58$, o indivíduo pode ser classificado como glaucoma. A sensibilidade nesse ponto é de $85,7 \%$, a especificidade $90,9 \%$, a acurácia $88,0 \%$ e a área abaixo da curva ROC de 0,920 (Figura 2).

\section{DISCUSSÃO}

No presente estudo, os valores de retardo da CFNR foram significantemente diferentes entre olhos normais e glaucomatosos. Excetuando-se o parâmetro simmetry, os valores das variáveis individuais foram estatisticamente diferentes, mesmo após a correção do erro alfa (Tabela 2). Entretanto, notamos considerável superposição entre os parâmetros isolados, mesmo entre aqueles com maior capacidade de discriminar entre olhos normais e glaucomatosos (Figura 1), ratificando a necessidade de se combinar 2 ou mais parâmetros para análise dos resultados do GDx para se diminuir eventuais resultados falso-positivos e falso-negativos. Nossos achados encontram-se concordantes com estudos prévios que mostraram a grande variabilidade individual dos parâmetros do GDx, e a menor diferenciação entre olhos normais e glaucomatosos pelo parâmetro simmetry ${ }^{(6-7,19)}$.

Os parâmetros individuais com melhor capacidade de discriminar entre olhos normais e glaucomatosos foram the number (Se: 79,5\%, Es: 81,8\%, área abaixo da curva ROC: 0,870), maximum modulation (Se: 83,0\%, Es: 76,1\%, área abaixo da curva ROC: 0,842) e ellipse modulation (Se: 65,2\%, Es: 88,6\%, área abaixo da curva ROC: 0,831) (tabela 3). O parâmetro the number consiste da análise de cerca de 130 variáveis através de um complexo cálculo neural de propagação retró$\operatorname{grada}^{(17)}$. Seus resultados são expressos na forma de um número que varia de 0 a 100, com números mais elevados indicativos de glaucoma. É o parâmetro individual que apresenta os melhores resultados discriminatórios, fato consistente com estudos anteriores envolvendo pacientes glaucomatosos com dano mais avançado (área abaixo da curva ROC: 0,870)(7), moderado (área abaixo da curva ROC: 0,780)(6) ${ }^{(6)}$ inicial (área

\begin{tabular}{|c|c|c|c|}
\hline & Normais & Glaucoma & p \\
\hline Idade (anos)* & 47 & 63 & $<0,001$ \\
\hline Raça (branca/negra/asiática)\# & 62 / 24 / 2 & $74 / 34 / 4$ & 0,75 \\
\hline Sexo (masculino/feminino) ${ }^{* *}$ & $35 / 53$ & $56 / 56$ & 0,14 \\
\hline Equivalente esférico (dioptrias) ${ }^{\dagger}$ & $0,32 \pm 0,92$ & $-0,3 \pm 2,34$ & 0,01 \\
\hline Olho (direito/esquerdo) ${ }^{\star *}$ & 49 / 39 & $64 / 48$ & 0,95 \\
\hline Mean Deviation $(\mathrm{dB})^{\dagger}$ & $-0,84 \pm 1,27$ & $-10,63 \pm 7,68$ & $<0,001$ \\
\hline Correct Pattern Standard Deviation $(\mathrm{dB})^{\dagger}$ & $1,60 \pm 0,99$ & $5,50 \pm 3,09$ & $<0,001$ \\
\hline
\end{tabular}

\begin{tabular}{|c|c|c|c|}
\hline GDx & Normais & Glaucoma & $p^{*}$ \\
\hline Symmetry & $0,95 \pm 0,15$ & $0,92 \pm 0,10$ & 0,4 \\
\hline Superior Ratio & $2,16 \pm 0,49$ & $1,61 \pm 0,36$ & $<0,001$ \\
\hline Inferior Ratio & $2,30 \pm 0,55$ & $1,75 \pm 0,40$ & $<0,001$ \\
\hline Superior/Nasal & $1,85 \pm 0,30$ & $1,47 \pm 0,28$ & $<0,001$ \\
\hline Maximum Modulation & $1,42 \pm 0,47$ & $0,83 \pm 0,38$ & $<0,001$ \\
\hline Ellipse Modulation & $2,67 \pm 0,71$ & $1,69 \pm 0,74$ & $<0,001$ \\
\hline The Number & $24 \pm 17$ & $58 \pm 25$ & $<0,001$ \\
\hline Average Thickness $(\mu \mathrm{m})$ & $65,12 \pm 13,86$ & $60,46 \pm 13,33$ & 0,02 \\
\hline Ellipse Average $(\mu \mathrm{m})$ & $68,38 \pm 14,45$ & $61,24 \pm 13,79$ & $<0,001$ \\
\hline Superior Average $(\mu \mathrm{m})$ & $75,18 \pm 18,86$ & $63,41 \pm 15,77$ & $<0,001$ \\
\hline Inferior Average $(\mu \mathrm{m})$ & $80,57 \pm 16,37$ & $69,91 \pm 16,04$ & $<0,001$ \\
\hline Superior Integral & $0,21 \pm 0,05$ & $0,19 \pm 0,05$ & 0,006 \\
\hline
\end{tabular}




\begin{tabular}{|c|c|c|c|c|}
\hline GDx & Ponto de corte & Sensibilidade (\%) & Especificidade (\%) & ROC \\
\hline Symmetry & $\leq 0,99$ & 77,7 & 39,8 & 0,533 \\
\hline Superior Ratio & $\leq 1,86$ & 81,3 & 69,3 & 0,816 \\
\hline Inferior Ratio & $\leq 1,93$ & 74,1 & 78,4 & 0,798 \\
\hline Superior/Nasal & $\leq 1,65$ & 75,9 & 79,5 & 0,818 \\
\hline Maximum Modulation & $\leq 1,11$ & 83,0 & 76,1 & 0,842 \\
\hline Ellipse Modulation & $\leq 1,84$ & 65,2 & 88,6 & 0,831 \\
\hline The Number & $\geq 34,5$ & 79,5 & 81,8 & 0,870 \\
\hline Average Thickness $(\mu \mathrm{m})$ & $\leq 53,5$ & 33,0 & 84,1 & 0,596 \\
\hline Ellipse Average $(\mu \mathrm{m})$ & $\leq 57,5$ & 45,5 & 81,8 & 0,653 \\
\hline Superior Average $(\mu \mathrm{m})$ & $\leq 61,5$ & 52,7 & 75,0 & 0,687 \\
\hline Inferior Average $(\mu \mathrm{m})$ & $\leq 71,5$ & 58,0 & 76,1 & 0,689 \\
\hline Superior Integral & $\leq 0,23$ & 85,7 & 35,2 & 0,613 \\
\hline
\end{tabular}

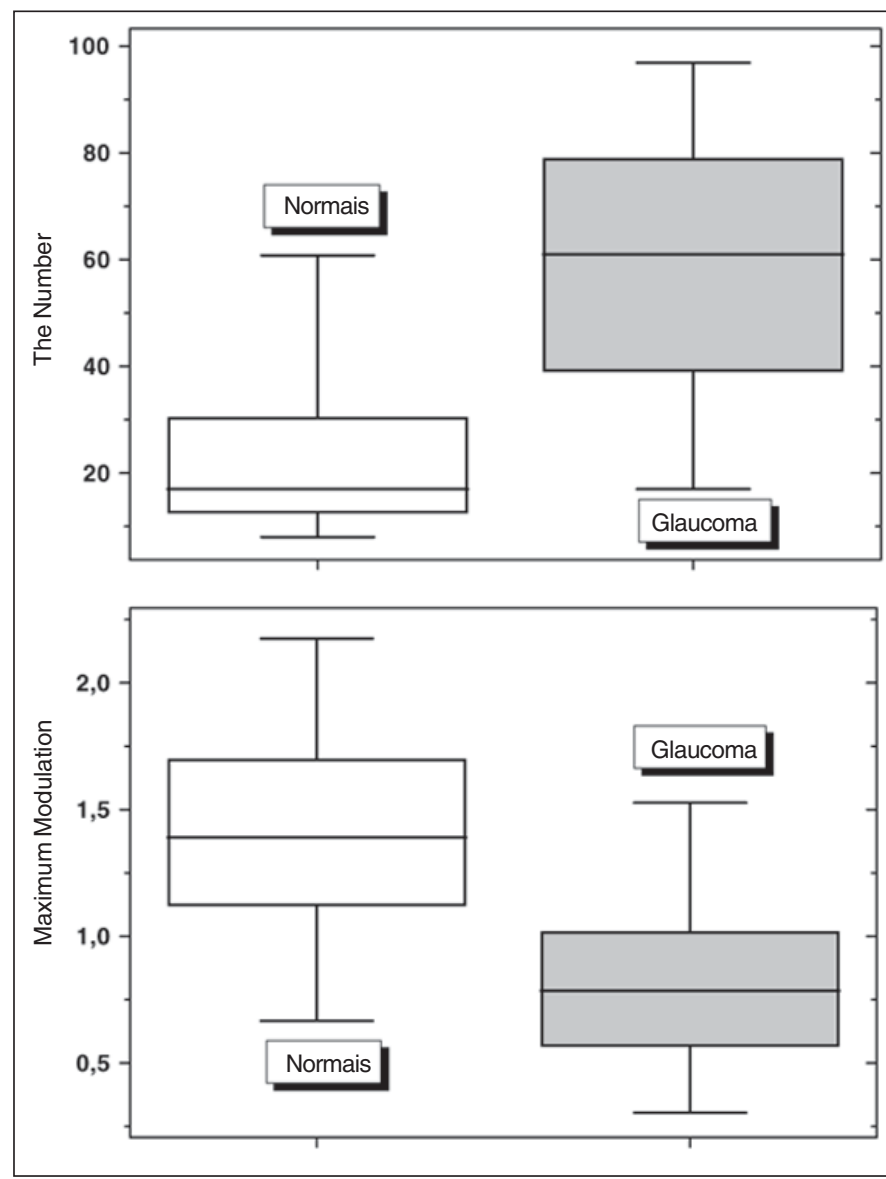

Figura 1 - "Box-Plot" demonstrando superposição entre olhos normais e glaucomatosos para os 2 principais parâmetros individuais do GDx

abaixo da curva ROC: 0,810) ${ }^{(19)}$. As modulações indicam a diferença ou variabilidade das medidas, com maior ou menor retardo nos diversos quadrantes (maximum modulation) ou dentro da elipse (ellipse modulation) ${ }^{(17)}$. Tendem a diminuir com a progressão do glaucoma e são fortes indicativos da lesão glaucomatosa, fato consistente em estudo anterior ${ }^{(7)}$,

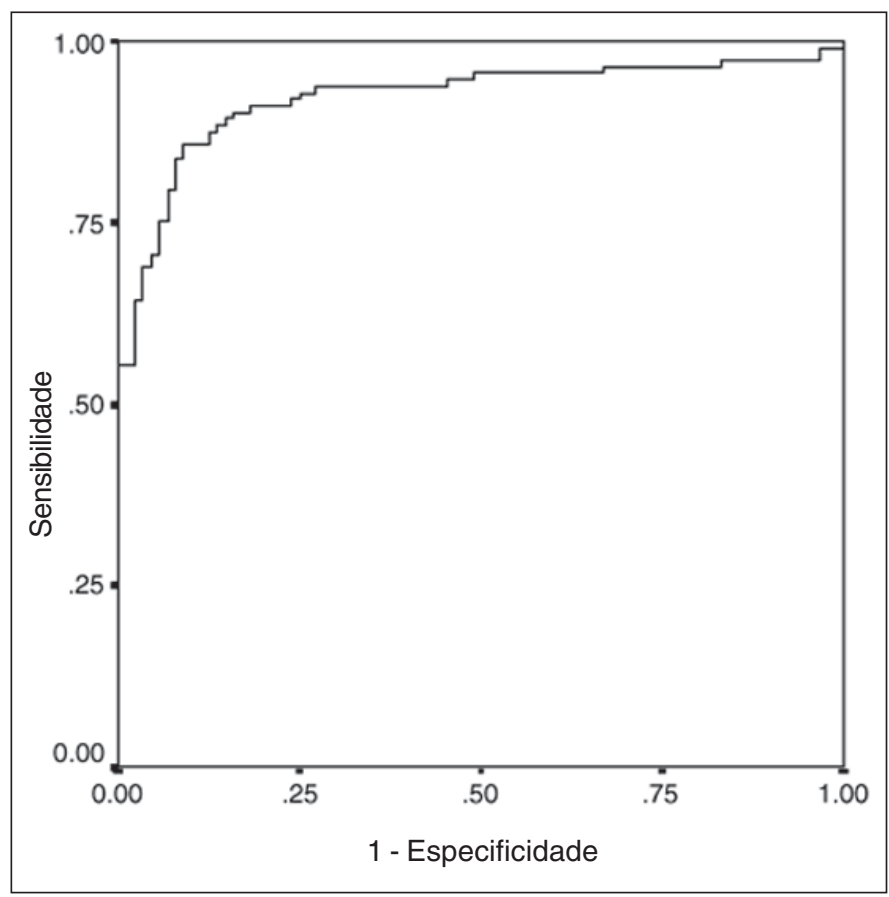

Figura 2 - Curva ROC para a análise logística multivariada com os parâmetros do GDx

Lauande-Pimentel et al. estudando pacientes normais e glaucomatosos (MD médio: -11,09 $\pm 9,10$ ) encontraram que os parâmetros individuais com melhor capacidade de discriminar entre olhos normais e glaucomatosos foram: the number, superior/nasal, ellipse modulation e maximum modulation, respectivamente ${ }^{(7)}$.

De acordo com o banco de dados do GDx, as variáveis isoladas do aparelho apresentam baixa sensibilidade e alta especificidade no diagnóstico do glaucoma. No nosso estudo, bem como em publicações anteriores ${ }^{(7,19)}$, houve uma nova padronização de novos pontos de corte para cada parâmetro isolado da polarimetria de varredura a laser, melhorando a relação Se/Es. Em uma doença que acarreta danos irreversíveis como o glaucoma, uma sensibilidade alta seria desejável 
para diminuir os resultados falso-negativos, e com isso um maior número de pacientes em fases iniciais do glaucoma poderiam se beneficiar do tratamento precoce. Entretanto, a especificidade deve ficar em níveis aceitáveis, semelhantes à sensibilidade para que o número de indivíduos tratados, mas sem glaucoma, seja o menor possível.

A criação de uma função discriminante incluindo idade, ellipse modulation e the number resultou em uma área abaixo da curva ROC $(0,920)$, sensibilidade (85,7\%) e especificidade (90,9\%) maior que qualquer parâmetro isolado do GDx (Tabela 3). A idade foi incluída como uma possível variável por causa da diferença estatisticamente significante entre os grupos. Weinreb et al. utilizaram os parâmetros average thickness, ellipse modulation e ellipse average para a melhor combinação de variáveis em uma fórmula discriminante de Fisher, obtendo uma área abaixo da curva ROC de 0,910 ${ }^{(6)}$. Entretanto, os autores puderam verificar que várias fórmulas discriminantes com a inclusão de diferentes parâmetros obtiveram resultados satisfatórios e muito semelhantes. Empregando o mesmo método, e os parâmetros ellipse modulation, the number, average thickness e ellipse average, Lauande-Pimentel et al. obtiveram uma área abaixo da curva ROC de 0,930 , sensibilidade de $90,4 \%$, especificidade de $82,4 \%$ e acurácia de $85,9 \%$ no diagnóstico do glaucoma com a polarimetria de varredura a laser ${ }^{(7)}$. Outros autores também propuseram fórmulas discriminantes lineares para o GDx ${ }^{(19-20)}$.

A diferença dos resultados obtidos por cada grupo pode ser explicada pelos critérios de inclusão e características da população glaucomatosa. Apesar de alguns especialistas considerarem a inclusão de características do disco óptico como viés na seleção de pacientes glaucomatosos em protocolos de imagem ${ }^{(21)}$, nós optamos por assim fazê-lo para diminuir a inclusão de pacientes suspeitos no grupo normal baseando-se apenas no exame de campo visual, um exame que apenas é alterado tardiamente no glaucoma ${ }^{(1-2)}$. Além do mais, em estudos anteriores com o GDx, a aparência do disco óptico foi utilizada como critério de inclusão ${ }^{(5-7,19)}$.

Concluindo, a combinação de dois ou mais fatores em uma função discriminante aumenta a capacidade da polarimetria de varredura a laser de discriminar entre olhos normais e glaucomatosos. Além disso, estamos oferecendo um novo método (análise logística multivariada) com eficácia semelhante à função linear. Entretanto, como a fórmula por nós criada foi testada na mesma população de onde foi derivada, e porque a nossa população glaucomatosa apresentava dano glaucomatoso moderado, estudos futuros com outras amostras e com dano glaucomatoso inicial são necessários para confirmar a aplicabilidade dos nossos achados.

\section{AGRADECIMENTOS}

Os autores gostariam de agradecer à Cleide M. Silva pelo auxílio estatístico.

\begin{abstract}
Purpose: To test the ability of the scanning laser polarimetry to discriminate between normal and glaucomatous eyes. Methods: One-hundred and twelve patients with primary open-angle glaucoma and 88 normal individuals were enrolled in the study. All individuals underwent a complete ophthalmic evaluation, a 24-2 full threshold Humphrey visual field and a GDx examination. Cutoff points were selected, ROC curves were created, and the sensitivity (Se) and specificity (Sp) were calculated for each individual GDx parameter. Finally, a multivariate logistic analysis was developed in order to achieve a better Se/Sp ratio for the diagnosis of glaucoma. Results: The best GDx individual parameters were: the number (Se: 79.5\%\%, Sp: $81.8 \%$, area under the ROC curve: 0.870 ), maximum modulation (Se: $83.0 \%$, Sp: 76.1\%, area under the ROC curve: 0.842) and ellipse modulation (Se: 65.2\%, Sp: 88.6\%). The multivariate logistic analysis resulted in an area under the ROC curve of 0.920 (Se: $85.7 \%$, Sp: $90.9 \%$ ). Conclusions: The combination of 2 or more parameters in a multivariate logistic analysis increases the ability of scanning laser polarimetry to discriminate between normal and glaucomatous eyes.
\end{abstract}

Keywords: Optic disk; Glaucoma/diagnosis; Lasers; Diagnostic techniques, ophthalmological

\section{REFERÊNCIAS}

1. Kerrigan-Baumrind LA, Quigley HA, Pease ME, Kerrigan DF, Mitchell RS. Number of ganglion cells in glaucoma eyes compared with threshold visual field tests in the same persons. Invest Ophthalmol Vis Sci 2000;41:741-8.

2. Quigley HA, Addicks EM, Green WR. Optic nerve damage in human glaucoma. III. Quantitative correlation of nerve fiber loss and visual field defect in glaucoma, ischemic neuropathy, papilledema, and toxic neuropathy. Arch Ophthalmol 1982;100:135-46.

3. Sommer A, Katz J, Quigley HA, Miller NR, Robin AL, Richter RC, Witt KA. Clinically detectable nerve fiber atrophy precedes the onset of glaucomatous field loss. Arch Ophthalmol 1991;109:77-83.

4. Quigley HA, Katz J, Derick RJ, Gilbert D, Sommer A. An evaluation of optic disc and nerve fiber layer examinations in monitoring progression of early glaucoma damage. Ophthalmology 1992;99:19-28.

5. Weinreb RN, Shakiba S, Zangwill L. Scanning laser polarimetry to measure the nerve fiber layer of normal and glaucomatous eyes. Am J Ophthalmol 1995;119:627-36.

6. Weinreb RN, Zangwill L, Berry CC, Bathija R, Sample PA. Detection of glaucoma with scanning laser polarimetry. Arch Ophthalmol 1998;116:1583-9.

7. Lauande-Pimentel R, Carvalho RA, Oliveira HC, Gonçalves DC, Silva LM, Costa VP. Discrimination between normal and glaucomatous eyes with visual field and scanning laser polarimetry measurements. $\mathrm{Br} \mathrm{J}$ Ophthalmol 2001;85:586-91.

8. Choplin NT, Lundy DC. The sensitivity and specificity of scanning laser polarimetry in the detection of glaucoma in a clinical setting. Ophthalmology 2001;108:899-904.

9. Kamal DS, Bunce C, Hitchings RA. Use of the GDx to detect differences in retinal nerve fiber layer thickness between normal, ocular hypertensive and early glaucomatous eyes. Eye 2000;14 (Pt 3A):367-70.

10. Colen TP, Tjon-Fo-sang MJ, Mulder PG, Lemij HG. Reproducibility of measurements with the nerve fiber analyzer (NfA/GDx). J Glaucoma 2000;9:363-70.

11. Almeida PB, Almeida GV, Cohen R, Prata Júnior JA, Melo PAA. Correlação e correspondência topográfica entre espessura da camada de fibras nervosas da retina e campo visual no glaucoma primário de ângulo aberto. Arq Bras Oftalmol 2001;64:109-15. 
12. Weinreb RN, Shakiba S, Sample PA, Shahrokni S, van Horn S, Garden VS et al. Association between quantitative nerve fiber layer measurement and visual field loss in glaucoma. Am J Ophthalmol 1995;120:732-8.

13. Caprioli J. Discrimination between normal and glaucomatous eyes. Invest Ophthalmol Vis Sci 1992;33:153-9.

14. Anderson DR, Patella VM. Automated static perimetry. St. Louis: Year Book Mosby; 1999.

15. Chylack LT Jr, Wolfe JK, Singer DM, Leske MC, Bullimore MA, Bailey IL et al. The lens opacities classification system III. The Longitudinal Study of Cataract Study Group. Arch Ophthalmol 1993;111:831-6.

16. Hodapp E, Parrish II RK, Anderson DR. Clinical decisions in glaucoma. St. Louis: Year Book; 1993.

17. Lauande-Pimentel R, Costa VP. Análise da camada de fibras nervosas da retina. Um guia para interpretar o exame de polarimetria. Rio de Janeiro: Cultura Médica; 2001.

18. Cross EM, Chaffin WW. Use of the binomial theorem in interpreting results of multiple tests of significance. Educat Psychol Measure 1982;42:25-34.

19. Zangwill LM, Bowd C, Berry CC, Williams J, Blumenthal EZ, SanchezGaleana CA et al. Discriminating between normal and glaucomatous eyes using the Heidelberg retina tomograph, GDx nerve fiber analyzer, and optical coherence tomograph. Arch Ophthalmol 2001;119:985-93.

20. Greaney MJ, Hoffman DC, Garway-Heath DF, Nakla M, Coleman AL, Caprioli J. Comparison of optic nerve imaging methods to distinguish normal eyes from those with glaucoma. Invest Ophthalmol Vis Sci 2002;43:140-5.

21. Garway-Heath DF, Hitchings RA. Sources of bias in studies of optic disc and retinal nerve fiber layer morphology. Br J Ophthalmol 1998;82:986.

\section{Congresso da Associação Centro Oeste de Oftalmologia}

\section{Congresso da Sociedade Brasiliense de Oftalmologia}

Simpósio da Sociedade Centro-Oeste de Glaucoma

\section{4 e 15 de Maio de 2004 Academia de Tênis de Brasília}

INFORMAÇÕES: Tels.: (61) 248-4030/81 16-3304 - Dr. Hilton Medeiros

(61) 248-4030/346-6971 (Ana Paula) - Dr. Geraldo Magela Vieira 